\title{
LA EFECTIVIDAD DE LOS DERECHOS SOCIALES EN SALUD PÚBLICA
}

CARLOS NETO, Daniel ${ }^{1}$

DENDASCK, Carla ${ }^{2}$

OLIVEIRA, Euzébio de ${ }^{3}$

CARLOS NETO, Daniel; DENDASCK, Carla; OLIVEIRA, Euzébio de. La efectividad de los derechos sociales en la salud pública. Conocimiento de base científica multidisciplinar revista Vol. 1. Año. 1. Marzo. 2016, pp: 3-14 ISSN:0959-2448

\section{RESUMEN}

Los derechos fundamentales han sido motivo de diversos debates en los últimos años, causando académicos para dedicarse a investigar tanto con el aspecto de salud, a través de la ley, la eficacia real de las directrices de las organizaciones. Este artículo pretende hacer una breve reflexión acerca de la efectividad real de los derechos sociales en la salud pública brasileña, utilizando como base, un análisis exploratorio

\footnotetext{
${ }^{1}$ Abogado. De la medicina académica. Doctorado en salud pública. Executive Mba. Especializada en servicios de salud aplicada. Graduación en salud de la familia. Especialista en auditoría. Correo electrónico: autor@nucleodoconhecimento.com.brv 2 Doctorado en psicoanálisis, post doctorado en psicoanálisis clínico y Máster en bioética, Director del centro de investigación y desarrollo de estudios avanzados, entrenador y profesor de cursos MBA en compañía, Campinas y São Paulo-correo electrónico: autor@nucleodoconhecimento.com.br

${ }^{3}$ Biólogo. Master en biología. Doctor en Medicina Tropical enfermedades. Investigador del programa graduado del centro de Medicina Tropical de la UFPA. Profesor de posgrado nivel y postgrado e investigador de la Universidad Federal de Pará - UFPA. Correo electrónico: autor@nucleodoconhecimento.com.br
} 
de la elucidações por varios estudiosos que permean para corroborar esto. Traer una breve discusión del mínimo existencial.

Palabras clave: Derechos sociales. Salud pública. Efectividad de los derechos fundamentales.

\section{INTRODUCCIÓN}

Los derechos fundamentales pueden definirse como un conjunto de reglas, principios, derechos, deberes e institutos involucrados en la soberanía popular, que garantizan la convivencia pacífica, libre e igual, sin importar credo, raza, origen, color, estatus social o condición económica, todos basados en el principio de la dignidad humana. (GLÓBULOS, 2010)

Sin embargo, a José Afonso da Silva, si conceptualización de los derechos fundamentales se convierte en una tarea difícil antes de las varias transformaciones con el tiempo, vea:

La expansión y transformación de los derechos humanos fundamentales en la historia participan hace difícil para ellos establecer un concepto sintético.

Aumenta esta dificultad la circunstancia emplear varias expresiones para designar a ellos, tales como: derechos naturales, derechos humanos, derechos humanos, derechos individuales, derechos subjetivos públicos, las libertades fundamentales, las libertades civiles y los derechos fundamentales y los derechos humanos fundamentales. (SILVA, 2005, p. 179)

Los derechos sociales ya son derechos fundamentales del hombre, como está formulando Moraes (2014, p. 595):

Los derechos sociales son derechos fundamentales del hombre, porque se refieren a un conjunto de relaciones sociales, económicas o culturales que se desarrolla para la realización de la vida en todo su potencial, sin que el titular no podía llegar y disfrutar de los bienes que necesita. 
Resulta que la principal dificultad para hacer frente en este tema se refiere a la aplicabilidad y efectividad de estos derechos, porque como todos sabemos, la salud es positivada en la lista de los derechos sociales.

Por lo tanto, es importante mencionar el concepto del término "eficacia" como la realización concreta de la función social del derecho, como sigue:

La noción de eficacia, es decir, que especifica la eficacia, corresponde a que Kelsendistinguir el concepto de efectividad de la norma-interpretado como ser "la realidad de su ser efectivamente implementado y observó, el hecho de que un ser humano se realice como el estándar para verificar los hechos". Eficacia significa la realización del derecho, el desempeño de su función social. Ella representa la materialización, en el mundo de los hechos, los preceptos legales y simboliza el acercamiento, tan cerca como sea posible, entre la normativa y debe ser de la realidad social. (BARROSO, 2002, p. 236)

Acerca de la efectividad de los derechos sociales es necesario para comprender no sólo el estado de derecho en un sentido amplio, pero el mismo enfoque con otros sistemas, tales como: político, económico, social e histórico.

Es necesario también la duración y la eficacia de la norma, como sigue:

El normativismo distingue, con precisión, la duración de la eficacia. La lección de Kelsen es muy clara en este sentido. La duración de la norma, le pertenece al debeser y no a la orden de ser. Término significa que la existencia especifica de la norma; eficacia es el hecho de que la norma es efectivamente aplicada y seguida; la circunstancia de que una conducta humana como la norma se aplica a los hechos. (SILVA, 2005, p. 64)

Uno de los aspectos más relevantes que rodean la efectividad de los derechos sociales fundamentales con respecto a la manera que fueron dispuestos en el texto constitucional, considerado por muchos autores e incluso metodológicamente inadecuados. En este sentido, se afirma que: 
Los derechos sociales fundamentales en la Constitución de Brasil están lejos de formar un grupo homogéneo, ya que, en cuanto a su contenido y la forma de su reconocimiento, la Asamblea Constituyente no siguió no constituida filas o teoría especifica. En cambio, terminó creando un capítulo muy contradictorios con respecto a la relación interna de los derechos y garantías. Esto, sin duda, implica una serie de inconvenientes interpretativos, que afectan directamente a la efectividad de las disposiciones contenidas en la Constitución. (KRELL, 2002, p. 21)

Aunque existe esta confusión, para Ana Paula de Barcellos, la eficacia jurídica de la norma está directamente relacionada con la fundamentalidade social, como se indica a continuación:

El primer criterio que guía la identificación de las modalidades de eficacia jurídica las declaraciones reglamentarias que le conciernen pueden estilo de condición social fundamentalidade para él, que es su nivel de importancia o relevancia social. Este es el parámetro lógico que impulsa la política legislativa en General. El más fundamental para la sociedad es disciplinada por el dispositivo y, por tanto, los efectos que tiene la intención de producir, más coherente debe ser la eficacia legal asociada (...). (BARCELLOS, 2002, p. 136)

Eficacia jurídica, entiende "capacidad (potencial) de una norma constitucional para producir efectos jurídicos". (KRELL 2002, p. 39).

Para producir los efectos jurídicos deseados, la eficacia jurídica de los derechos sociales, están en plena vigencia las normas subdividen, efectivamente contienen y limitada eficacia, como se explica a continuación.

Estándares de eficiencia completo tienen aplicabilidad inmediata y así son independientes de la posterior legislación para su plena aplicación. Se afirma que:

Son aquellos que, desde la entrada en vigor de la Constitución, producir y posiblemente producir todos los efectos esenciales, en relación con los intereses, comportamientos y situaciones que el legislador constituyente, directa y normativamente, que pretende regular. (SILVA, 2005, p. 101) 
Normas constitucionales de eficacia ya son que tienen carácter obligatorio, pero también son limitantes del poder público, tal y como aparece:

Las normas de eficacia contenidas son aquellas que el legislador constituyente reguló suficientemente los intereses relativos a una materia particular, sino izquierda habitación la parte restrictiva de la competencia discrecional de las autoridades públicas, con arreglo a la ley establece o en términos de conceptos generales en él. (SILVA, 2005, p. 116)

Y por último pero no menos importante, las normas de eficacia limitada que se basan en ley para regularlos. Se llaman también "estándares de aplicación diferida, estándares de estándares de eficacia y eficiencia inmediata" (células de la sangre, 2010, p. 146)

Así que usted puede ver claramente que la aplicabilidad de los derechos sociales es idéntica a la de garantías y derechos fundamentales. Es decir, tener aplicabilidad inmediata como el §1 del arte. 5 de CF/1988.

\section{LA CUESTIÓN DE LA EFECTIVIDAD DE LOS DERECHOS SOCIALES EN LA CONSTITUCIÓN}

Mucho hablar de la doctrina sobre la eficacia y la aplicabilidad de los derechos constitucionales. Sin embargo, independientemente de la forma de reconocimiento, las normas de derechos fundamentales deben generar un mínimo de efectos jurídicos, porque como has visto, todo el estándar tiene eficacia y aplicabilidad.

Sin embargo, es que el grado de efectividad de los derechos sociales fundamentales se determinará por la forma de la Constitución y reconocimiento de las peculiaridades de su objeto. (SARLET, 2007, 237-238)

El mayor problema actualmente no se refiere a los derechos, sino por la falta de eficacia de las normas constitucionales, ver: 
El problema fundamental en relación con los derechos humanos, hoy en día, no es tanto para justificarlos, sino para proteger a (...). No es una cuestión de saber qué y el cómo son estos derechos, que la naturaleza y su fundación, ya sean natural o histórico de los derechos, absoluto o relativo, pero cuál es la manera más segura de garantizarlos, para impedir que, a pesar de las solemnes declaraciones que son continuamente violaron (BOBBIO, 1992, p. 24-25)

De esta manera, reducirá la eficacia de lo social fundamental de los derechos no solamente la ausencia de leyes ordinarias.

El mayor problema no es la prestación de los servicios sociales básicos del gobierno, ya que la gran mayoría de las normas para el ejercicio de los derechos sociales ya existe. Ver si el problema está en la "formulación, implementación y mantenimiento de sus políticas públicas y en la composición del gasto en los presupuestos de la Unión, los Estados y los municipios". (KRELL, 2002, p. 31-32)

Otro problema que se discute en la doctrina es en la determinación de los elementos que constituyen los derechos sociales fundamentales que conecta con el hecho de estos derechos sólo existen "cuando las leyes y las políticas sociales la garantía. En otras palabras: es el legislador ordinario que crea y determina el contenido de un derecho social "(CANOTILHO, 2002, p. 481).

Esta parte importante de la doctrina considera que la mayoría de las disposiciones constitucionales relativas a los derechos sociales "como no se puede presentar cualquier otro eficacia jurídica además de atar negativamente la legislatura actuar explícitamente contra el objetivo indicado por la norma". (BARCELLOS, 2002, p. 162)

Dicho esto, parece que gran parte de la doctrina suele clasificar definir estándares de derechos sociales como normas programáticas, ya que necesitan un logro legislativo que puede generar la plenitud de sus efectos.

En este sentido, se afirma que las normas programáticas son: 
En la legislatura, constituyente o no, en lugar de la edición de norma jurídica de aplicación práctica, sólo rastros de directores de líneas, que guiará a los poderes públicos. La legislación, la ejecución y la justicia estarán sujetos a estos dictados, que son como los datos de los programas a su función (BARROSO, 2002, p. 115)

Todavía, según Krell (2002, p. 27-28), las normas programáticas sirven como coartada para crear una imagen que el estado responde, normativamente, los problemas de la sociedad, ver:

Muchas normas constitucionales de derechos sociales programáticos, por no tener un mínimo de condiciones para su implementación, servir sólo una coartada para crear la imagen de un estado que responde normativamente los problemas reales de la sociedad, desempeñando una función principalmente ideológica en constituyen una forma de manipulación o ilusión que inmuniza el sistema político frente a otras alternativas.

Sin embargo, esto no parece ser la tendencia actual de la eficacia de los derechos sociales, frente a los argumentos, solo revelan el peligro si dejas la voluntad del legislador $\mathrm{o}$ el administrador determinar total efectos sociales de los derechos fundamentales.

Como Estados Barcellos (2002, p. 192): "esto podría causar un vacío real fundamentabilidade del principio de dignidad de la persona humana como un vector de interpretación constitucional."

Cabe destacar aquí, que la situación se vuelve más complicada donde el gobierno mantiene inerte, es decir, donde no todavía instalado los servicios necesarios o que funcionan mal.

Los beneficios positivos de los derechos sociales deben proporcionados por el estado y el apoyo de la sociedad, quien asumirá estos costos, recogidos a través de impuestos entre otros como apoyo: 
Definitivamente hay recursos, más claro y formas textuales precisas no serán capaces de superar esta realidad objetiva, será inviables reglas. Luís Roberto Barroso ya identificar esta situación, en el que la aparente ausencia de condiciones materiales condena la norma desde sus inicios, como una forma de falta de sinceridad. Por lo tanto, qué pasa si usted quiere enfatizar, es que al cuidar de la interpretación del derecho público en General, y del constitucional particular, es necesario tener en cuenta, además de los elementos puramente legales, datos de la realidad, uno de ellos es las condiciones materiales y alcanzar los controles regulatorios financieros. (BARCELLOS, 2002, p. 259-260)

Sin embargo, antes de que el mal funcionamiento o incluso falta de servicios esenciales para el bienestar de la población, "formulación de políticas públicas de protección de ciertas categorías sociales económicamente marginados y excluidos", estas políticas que demandan principalmente gestión del estado, siendo de suma importancia para su aplicación, las alianzas con la sociedad civil. (KRELL, 2002, p. 34)

Pronto, los derechos derivados de beneficios, derivados de las regulaciones de los derechos sociales establecidos en la Constitución, implica, que ya desplegó el servicio público es necesario para la satisfacción de un Fundamental derecho, que su no disposición en violación de la ley ordinaria puede, en Brasil, ser atacado por medio de amparo, instrumento jurídico eficaz en el control judicial de actos administrativos.

Así, se afirma que:

La eficacia que debe acompañar a la normativa de los derechos sociales fundamentales - especialmente cuando fundada en la dignidad de la persona humana, debe ser positivo o simétrico, porque permite que el poder judicial, si hay una violación inconstitucional, ejecución de la ley. Las modalidades de interpretación y eficacia negativo vedativas de marcha atrás, aunque absolutamente relevante y actual, son una protección insuficiente de ciertas variables fácticas derivadas del imperativo del respeto por el núcleo esencial de los derechos sociales. (BARKER, 2011, p. 102-103) 
Por ello abordar los problemas de efectividad de los derechos fundamentales sociales, allí es no hay manera de ignorar su función primaria de los derechos de disposición, así como su reconocimiento en el texto constitucional, puesto que ambos aspectos, todas las pruebas, constituyen factores estrechamente vinculados con el grado de efectividad y aplicabilidad de los derechos fundamentales, como ya se mencionó.

Otra cuestión que socava la efectividad de los derechos fundamentales es la denominada reserva 'posible', que se ha utilizado para indicar la limitación de los recursos antes de la necesidad de la garantía de los derechos fundamentales y sociales. Como lo demuestra Barcellos (2002, p. 261):

El debate sobre esta cuestión ha sido identificado en Brasil a través de la expresión posible reserva y popularizado en gran parte por el gobierno de compromiso con la promoción y sostiene en las más diversas demandas, con el pretexto de inminente Apocalipsis económico siempre.

Resulta que la escasez de recursos presupuestarios no puede ser obstáculos para la garantía de los derechos sociales, que son condiciones esenciales de la vida humana, porque de esta manera, en última instancia, violando preceptos básicos y fundamentales de la Constitución Federal: el principio de la dignidad humana.

De esta manera garantizar el mínimo existencial es requisito indispensable para la supervivencia del individuo, porque si no tienes las condiciones necesarias para una vida digna, las funciones sociales no están siendo eficaces. Y en cuanto al mínimo existencial para salud, viola el derecho a la vida, porque sin salud no se puede vivir.

\section{EL PRINCIPIO DE RESERVA POSIBLE}

Usted no cabe duda que la garantía legal de los derechos sociales no depende sólo de beneficios del estado, porque su eficacia depende de recursos del estado (Unión, estado, Distrito Federal y municipios), pero éstos se limitan. Por esta razón, la efectividad de estos derechos se limita a los recursos presupuestarios disponibles. Según Sarlet y boss (2007, p. 265): 
La colocación de los derechos sociales a prestaciones en virtud de lo que se llama una reserva posible que, entendida en un sentido amplio, que abarca tanto la posibilidad, como el poder de disposición por parte del destinatario de la norma.

Para divagar sobre el tema, es necesario primero para conceptualizar la posible reserva de Nota:

La reserva del término posible intenta identificar el fenómeno económico de la limitación de recursos disponibles en la necesidad casi siempre infinita de ellos. Lo importante para el estudio realizado, las posibilidades que, además de las discusiones legales sobre lo que puede ser esperado en la corte en el estado - y el último informe de la sociedad, por ello es que la sustenta - es importante recordar que hay un límite de posibilidades materiales de estos derechos. (BARCELLOS, 2002, p. 261-262)

Esta pregunta acerca de la disponibilidad efectiva de fondos es vista por la doctrina como una limitación objetiva para la efectividad de los derechos sociales. Sin embargo, también existe otra limitación que es acerca de la posibilidad jurídica de que la disposición de recursos.

Sobre el tema, dijo Barcellos (2002, p. 262-263):

Bajo el título general de la reserva puede vivir por lo menos dos especies diferentes de fenómenos. El primero de estos acuerdos con la ausencia de los recursos fácticos, algo cerca del presupuesto y el agotamiento puede ser identificado como una reserva de posibles hechos. Es posible cuestionar la realidad de este tipo de circunstancias cuando se trata de autoridades públicas, teniendo en cuenta la forma de recaudación de fondos y la naturaleza de las entradas. De todos modos, la falta de recursos describir situaciones sobre las cuales se podría hablar de posibles hechos reservados.

El segundo fenómeno identifica una posible reserva legal puesto que no describe exactamente un estado de agotamiento de los recursos y la ausencia de autorización del presupuesto para ciertos gastos en particular. 
Pronto, el principio de la reserva es una limitación de hecho y de derecho que el estado va ayuda para proveer a quien corresponda los derechos sociales. Este es el gran problema aquí planteado, porque se refiere a múltiples reclamaciones por el gobierno de este principio, sólo para librarse de la obligación de aplicación de los derechos fundamentales.

En relación con tal problema se indica:

El Brasil tiene uno de los peores cuadros de distribución del ingreso en el mundo, y condición de la realización de los derechos económicos, sociales y culturales a la existencia de los recursos disponibles en el estado, significa reducir su eficacia a cero, pone su universalidad, condenando a considerar los derechos de menores (KRELL, 2002, p. 54)

Es decir, la mayoría de la población brasileña que es pobre, es decir, deficiencia en los servicios sociales que más sufre de este choque de ser tener la ejecución de derechos basados en la disponibilidad de los recursos públicos.

Por lo tanto, aunque la efectividad de los derechos sociales dependen de la consideración del gobierno en sus decisiones frente a la escasez de recursos, la misma no puede eludir su deber constitucional de proporcionar y garantizar los derechos mínimos a la población para una vida con dignidad y en ausencia de cualquiera de ellos, es hasta el poder judicial, a través de la provocación resolver cualquier problema sobre el tema y que ha ocurrido con la frecuencia que muestra el análisis de problemas.

\section{CONSIDERACIONES FINALES}

Suponiendo que las necesidades de la población son infinitas y recursos limitados, debe tenerse en cuenta acerca de cómo establecer prioridades en la asignación de recursos para garantizar el mínimo establecido en la Constitución Federal.

En primer lugar, señalar que la formulación del concepto existencial mínima que se originó en el Tribunal Constitucional alemán, que el derecho a un mínimo de existencia 
del principio de dignidad humana (Constitución, arte. 1, I) y del derecho a la vida y la integridad física, a través de la interpretación sistemática por el principio del Estado Social (LF, arte. 20, I) (KRELL.2002.

Como complemento de la comprensión:

Al principio, la calificación, calificación de la dignidad humana como principio fundamental expresar la certeza de que el arte. $1^{\circ} \stackrel{\circ}{ }$ Inc. III, de nuestra ley fundamental no contiene sólo una declaración de contenido ético y moral (que she, pero en definitiva, tiene), sino que constituye la norma legal-positivo con rango constitucional y, como tal, con eficacia, convirtiendo tal suerte, más allá de la dimensión ética, ya señalada, en valor jurídico de la comunidad. En este contexto, deben considerarse, siempre que el principio fundamental, la dignidad de la persona humana no es sólo guía el valor de los derechos fundamentales y del orden constitucional general, que justifica plenamente su caracterización como un principio constitucional de mayor axiológica jerarquía valorativa. (SARLET, 2007, p. 111-112)

Es decir, el mínimo existencial es vinculado a la dignidad de la persona humana y debe ser protegido por los derechos sociales de características con el fin de proveer condiciones mínimas de la población.

En un intento por delimitar el contenido del mínimo existencial, una posible solución es el uso del principio de dignidad de la persona humana para asegurar las condiciones mínimas de la población para una vida sana, sugiriendo cómo mínimo parámetros guía establecido por la Organización Mundial de la salud. (SARLET, 2006, p. 47-59)

Sin embargo, hay todavía gran sala política del legislador, puesto que no hay ningún IE "una medida correcta y una manera única de conformidad con el imperativo constitucional": existe un contenido fijo del derecho a una existencia decente mínimopara, que sólo se puede definir en el caso concreto. (ANDRADE, 2002, p. 27) 
De todo tipo, el valor predeterminado mínimo de supervivencia debe incluir siempre una salud básico y eficiente, acceso a alimentos básicos y ropa, la educación de primer grado y la garantía de una vivienda, como a continuación:

Ahora, si la empresa no es capaz de reconocer en qué punto se encuentra en una situación indigna, es decir, si no existe un consenso sobre el contenido mínimo de dignidad, "se enfrentarán a una crisis ética y moral de tales proporciones que el principio de la dignidad humana se transformará en una fórmula totalmente vacía" (BARCELLOS 2002, p. 197)

Pronto, el mínimo social garantizado en las condiciones materiales de existencia se basa en el concepto de la dignidad humana y la retórica de los derechos sociales mínimos de este depreciaria, sino más bien fortalecería en su esencial dimensión como expresión de la ciudadanía reivindicatoria.

Garantizada constitucionalmente, debe ser reparado el mínimo existencial como una prioridad, dice Barcellos (2002, p. 268):

Si eso es así y significa que los recursos financieros no son ilimitados, los recursos disponibles deben aplicarse como una prioridad en el servicio de los propósitos considerados esenciales por la Constitución hasta que se alcanzan.

Los recursos restantes será diseñado de acuerdo a las opciones políticas que deliberación democrática para establecer en cada momento.

Mientras que el mínimo existencial, inherente constitucionales garantiza las prioridades (características de los derechos fundamentales), tiene aplicabilidad inmediata por ser en los derechos fundamentales independientemente de si o no social. Por otro lado, como la fuente no se ocupa de los derechos sociales vinculados al mínimo existencial, estará condicionada a las limitaciones de los recursos presupuestarios.

Sin embargo, es las autoridades públicas el deber de cumplir con lo que la Constitución garantiza como Krell (2002, p. 60): 
Donde el estado crea estas ofertas para el colectivo, se debe garantizar la posibilidad de participación ciudadana. Y si la legislación no concede un derecho a expresar individuales reciben servicios vitales, el ciudadano puede apelar al derecho fundamental de igualdad en relación con el principio del Estado Social

Una vez violado el mínimo existencial, por la omisión de la eficacia de lo social fundamental de los derechos, el derecho a demandar en la corte.

La razón que justifica tal judicialización de los derechos sociales, inherentes al mínimo existencial, en virtud de que tales derechos son indispensables para tener una vida con dignidad, que es la teoría de la reserva no puede enviar aquí. Aunque judicialización tratando de disminuir este problema de la falta de recursos, tienes que tener una visión en que la finalidad esencial previsto en la Constitución, y si los recursos son suficientes para satisfacer la aplicación prevista. (BARKER, 2011).

Pero se enfrenta con el mínimo existencial que evaluará las prioridades de gasto público, basado en la Constitución y también puede definir las actividades jurisdiccionales de los derechos sociales

Según Canotilho (2002), el estado debe velar por la efectividad de los derechos sociales como ya sabes, pero vale la pena decir, también, que el mismo no puede reducir, cancelar o revocar lo que hoy entendemos como mínimo existencial y deberá sellar todas las medidas legislativas encaminadas a esto. Este es el principio de prohibición de la reacción social.

Conceptualización-if al revés dice:

Al revés, por último, es una derivación de la eficacia negativa, particularmente vinculada a los principios que involucran derechos fundamentales. Asume que estos principios se implementan a través de las normas infra-constitucionales y, basado en el derecho constitucional vigente, a efectos generales por estos principios es la expansión progresiva de los derechos fundamentales. A partir de estos supuestos, que al contrario puede requerir del poder judicial propone es la invalidez de la revocación de la norma que, por el principio, para otorgar o extender los derechos 
fundamentales, sin que la revocación de que se trate se acompañada de un equivalente o sustituto de la política de regulación. (BARROSO, 2002, p. 379)

Por lo tanto se concluye que este sello es una manera de prevenir la derogación de las leyes que garantizan los derechos sociales, no a disminuir lo que se logró a través de la evolución histórica, tales como el establecimiento de normas mínimas para poder tener una vida con dignidad.

De esta manera, el papel del poder judicial en materia de derechos sociales es importante porque es el control de los derechos sociales fundamentales, análisis de la esencialidad de la demanda y el grado de necesidad según cada caso individual. Y que estos judicialización de los derechos sociales y sobre todo salud, objeto del presente estudio, se denomina la judicialización del fenómeno salud.

\section{REFERENCIAS BIBLIOGRÁFICAS}

ALMA-ATA. Alma Ata. 1978. Disponible en: http://www.saudepublica.web.pt/05promocaosaude/Dec_Alma-Ata.htm. Acceso en: nov 09. 2015.

ANDRADE, José Carlos Vieira de. El poder judicial y los derechos sociales fundamentales: acceso a la salud, la polémica cuestión. Porto Alegre: Tribunal de justicia, 2002.

BARCELLOS, Emanuel. La eficacia jurídica de los principios constitucionales: el principio de la dignidad humana. Rio de Janeiro: Renovar, 2002.

BARROSO, Luis Roberto. El derecho constitucional y la eficacia de sus normas: límites y posibilidades de la Constitución brasileña. ed 6. São Paulo: Renovar, 2002

BARKER, Ramon F. Judicialización del derecho a la salud: la tensión entre el "mínimo existencial" y la "reserva posible" en la búsqueda de la preservación de la dignidad humana. Curitiba: Juruá Editora, 2011. 
Brasil. Constitución (1988). Constitución de la República Federativa de Brasil. Brasilia. DF: Senado, 2014.

Brasil. Ley no. 8.080 de 19 de septiembre de 1990. Normas sobre las condiciones de la promoción, protección y recuperación de la salud, organización y funcionamiento de los servicios correspondientes y otros asuntos. Diário Oficial da União, ejecutivo, Brasília, DF.

CÉLULAS sanguíneas, Uadi Lammego. Derecho constitucional al alcance de todos. 2 o Ed. São Paulo: Saraiva, 2010.

CANOTILHO, José Joaquim Gomes. Derecho constitucional y teoría de la Constitución. 6. ed. Coimbra: Almedina, 2002.

KRELL, Joachim de Andrea. Los derechos sociales y el Control Judicial en Brasil y en Alemania: los caminos de la (ONU) de un derecho constitucional comparado. Porto Alegre: Sergio Antonio Fabris, 2002

Moraes, Guilherme pena. Curso de derecho constitucional. São Paulo: Atlas, 2014.

SARLET, Ingo Wolfgang. La efectividad de los derechos fundamentales. 7. Ed. Porto Alegre: Editora abogado, 2007.

. Dignidad humana y derechos fundamentales en la Constitución Federal de 1988. ed 4. Porto Alegre: Abogado de Editora, 2006.

SILVA, José Afonso da. Comentario contextual a la Constitución. São Paulo: Malheiros, 2005. 